\title{
Atypical progression of multiple myeloma with extensive extramedullary disease
}

\author{
S N Jowitt, A Jacobs, P A Batman, D A Sapherson
}

\begin{abstract}
Multiple myeloma is a neoplastic disorder caused by the proliferation of a transformed B lymphoid progenitor cell that gives rise to a clone of immunoglobulin-secreting cells. Other plasma cell tumours include solitary plasmacytoma of bone (SPB) and extramedullary plasmacytomas (EMP). Despite an apparent common origin there exist pathological and clinical differences between these neoplasms and the association between them is not completely understood. A case of IgG multiple myeloma that presented with typical clinical and laboratory features, including a bone marrow infiltrated by well differentiated plasma cells, is reported. The tumour had an unusual evolution, with the development of extensive extramedullary disease while maintaining mature histological features.
\end{abstract}

(F Clin Pathol 1994;47:269-271)

\section{Case report}

A 67 year old man of Indian descent presented with a three month history of low back pain. Physical examination was unremarkable. Plain films of the lumbar spine revealed a lytic lesion in the vertebral body of L3 associated with end plate collapse; lateral views showed an abnormal soft tissue mass anterior to the same vertebral body. A skeletal survey identified multiple lucent areas in the skull vault but no additional lytic lesions were seen elsewhere. The diagnosis of multiple myeloma was confirmed by the following investigations: electrophoresis revealed an IgG $\lambda$ paraprotein (quantitated at $50 \mathrm{~g} / \mathrm{l}$ ) in the serum and free $\lambda$ light chains in the urine; a bone marrow aspirate contained $27 \%$ myeloma cells in the form of well differentiated plasma cells.

After receiving local radiotherapy to the lumbar spine the patient was given melphalan $(10 \mathrm{mg} /$ day for seven days) and prednisolone (40 $\mathrm{mg} /$ day for seven days), repeated at monthly intervals. The plateau phase was reached after 12 courses of the aforementioned chemotherapy (paraprotein concentration $3 \mathrm{~g} / \mathrm{l}$ ) and treatment was discontinued.

Two months later the patient presented with central abdominal discomfort. Physical examination yielded normal results. An abdominal ultrasound scan showed an hypoechoic mass measuring $6 \mathrm{~cm}$ in diameter and situated anteriorly to the lumbar spine; normal kidneys and pancreas were identified.

Plain films confirmed that this was the same soft tissue mass identified at presentation and implied the mass represented soft tissue extension of myeloma. After four weeks of clinical observation the abdominal mass had become palpable and the serum paraprotein had risen to $13 \mathrm{~g} / \mathrm{l}$. The patient was again given melphalan (10 mg/day for five days) and prednisolone (40 $\mathrm{mg} /$ day for five days) and local radiotherapy to the abdominal mass. The mass became impalpable and after four further courses of chemotherapy the serum paraprotein had fallen to $4 \mathrm{~g} / \mathrm{l}$.

Six weeks after completion of the fifth course of melphalan and prednisolone the patient re-presented with abdominal pain and a mass was palpable in the right hand side of the abdomen. Investigations of note were as follows: haemoglobin concentration $88 \mathrm{~g} / \mathrm{l}$; white cell count $1.9 \times 10^{9} / 1$ (differential of neutrophils $1.3 \times 10^{9} / 1$, lymphocytes $0.4 \times$ $10^{9} / 1$, monocytes $0.2 \times 10^{9} / 1$ ); platelet count of $71 \times 10^{9} / 1$; paraprotein concentration $15 \mathrm{~g} / 1$; bone marrow aspirate was diluted with blood but a trephine biopsy specimen revealed a hypocellular marrow with reduced normal haemopoiesis and foci of plasma cells. Circulating plasma cells were not detected at any stage. An abdominal ultrasound scan detected a mass in the right para-aortic region and another anterior to the splenic vein; the right kidney also appeared to have been completely replaced by abnormal tumour tissue. An ultrasound guided biopsy specimen of the right renal mass was obtained, the histology of which showed sheets of plasma cells with areas of necrosis with no renal tissue identified. A computed tomogram of the abdomen was performed and relevant abnormalities are shown in fig 1 .

The serum paraprotein rose to a concentration of $19 \mathrm{~g} / 1$ over the course of one week and the patient's general condition began to worsen. Haemopoietic recovery was delayed and the patient was given methylprednisolone ( $1.5 \mathrm{~g} /$ day for five days). He developed symptoms and signs of pulmonary sepsis and was given broad spectrum antibiotics. Unfortunately, he continued to deteriorate and died 10 days later. Two days earlier his serum paraprotein had measured $31 \mathrm{~g} / 1$.

Post mortem examination confirmed the presence of bronchopneumonia together with evidence of disseminated tumour of the right kidney, pancreas, porta hepatis, serosa of the transverse colon, pelvic and left perirenal fat, left-sided ribs, and left parietal pleura.

Histological examination showed a monomorphic diffuse infiltrate of plasmacytoid cells with little supporting stroma 
Figure 1 Computed tomogram of abdomen and pelvis after intravenous contrast.

(A) At level L1 is an extensive retroperitoneal mass replacing the

pancreas and invading the right kidney. There are further masses anterior and posterior to the left kidney. (B) At the level of the renal hila are several masses in the base of the mesentery and almost no opacification of the right kidney, implying loss of function due to invasion. (C) At level L4 is a necrotic mesenteric mass and also several peritoneal deposits (arrows). (D) Several soft tissue masses (arrows) around the sigmoid colon in the pelvis.
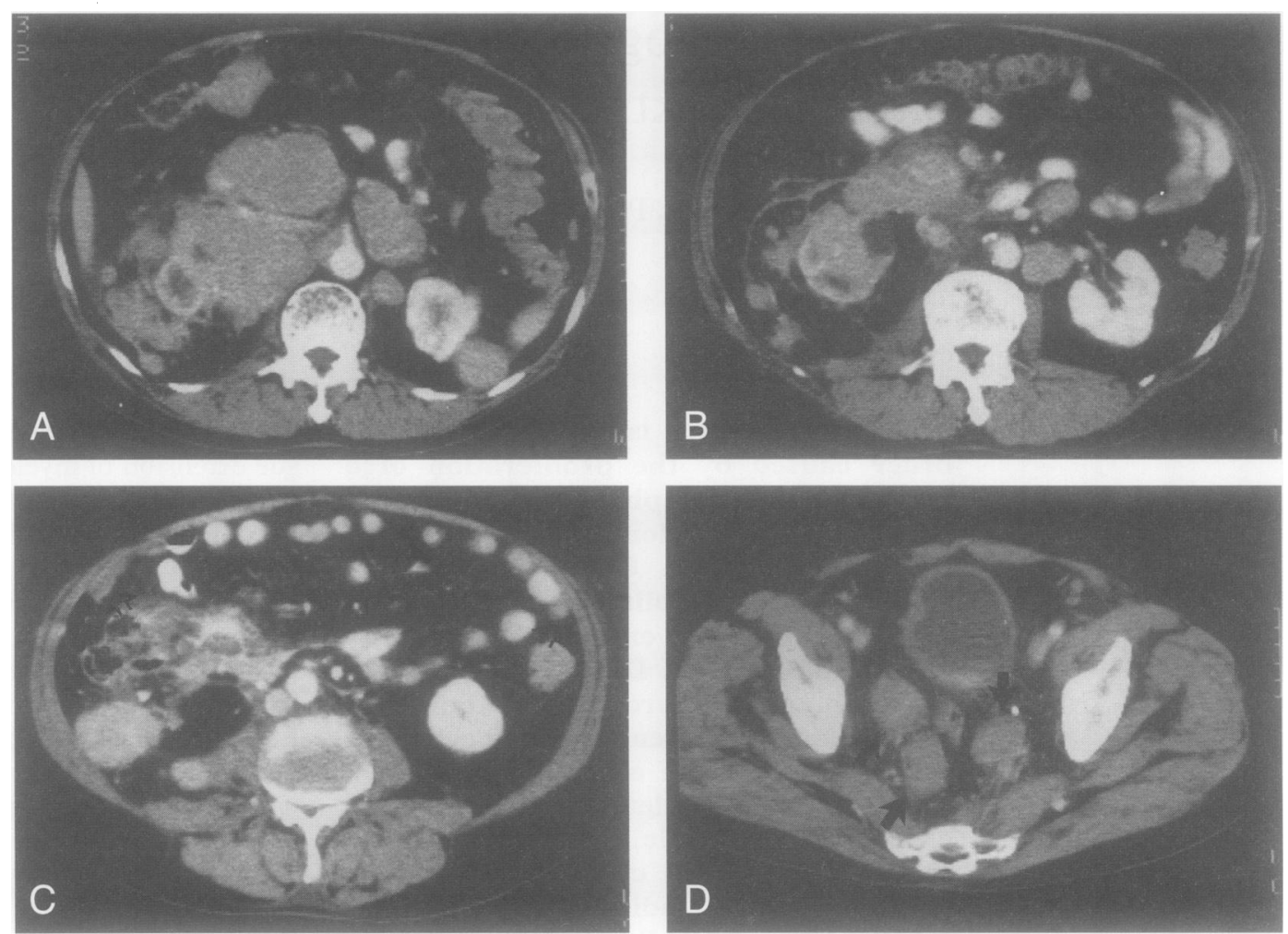

(fig 2A). The neoplastic cells showed typical plasma cell features with an eccentric nucleus and perinuclear hof. Immunoperoxidase staining showed $\lambda$ light chain restriction (fig $2 B$ ).

\section{Discussion}

We have described a case of multiple myeloma which, after an initial response to chemotherapy, progressed in an atypical manner, with the development of multiple extramedullary plasma cell tumours affecting the right kidney, pancreas, porta hepatis serosa of the transverse colon, pelvic and left peri-renal fat, left-sided ribs and left parietal pleura.

Multiple myeloma generally presents due to the result of dissemination of malignant plasma cells throughout the bone marrow and the effects of the excessive amount of monoclonal protein they produce. The primary focus of the tumour is not usually evident. It is not uncommon to see the effects of extraosseous extension of intramedullary tumour nodules as a result of erosion of the cortex and subsequent spread beyond the periosteum, although soft tissue involvement itself is unusual except in the rare IgD multiple myeloma. ${ }^{1}$ In the case we have described there was radiological evidence of spread from the L3 vertebral body, but no clinical evidence of any other soft tissue disease at presentation. Relapse from a plateau phase in multiple myeloma is most often manifest by impairment of bone marrow function together with the reappearance and gradual increase in the concentration of the paraprotein. In some instances locally aggressive tumours develop. These tend to arise in pre-existing bony lesions rather than in soft tissue sites.
Although microscopic disease of the spleen, liver, or lymph nodes is found in up to $70 \%$ of cases at post mortem examination the presence of gross extramedullary tumour formation at other locations is less common and rarely involves more than a single site. ${ }^{2}$ The pattern of spread shown in our case is very unusual for multiple myeloma. In an extensive literature search we have been able to find only one report of multiple myeloma with comparable features. Foucar described two cases of multiple myeloma that developed prominent extramedullary disease at multiple sites one and four years after initial diagnosis. ${ }^{3}$ both cases had anaplastic features and seemed to represent a variant of multiple myeloma which resulted from a transformation of well differentiated myeloma cells to poorly differentiated immunoblastic-like cells. In contrast, the multiple extramedullary tumours in our case were all composed of cells with features of plasmacytoid differentiation.

The association between the different categories of plasma cell tumours has been the subject of much debate. ${ }^{4-6}$ Solitary myeloma of bone (SMB) usually presents as a locally painful lesion with no evidence of widespread dissemination of plasma cells in the bone marrow, although in 10-20\% of cases monoclonal protein is detected. ${ }^{5}$ However, there is a high rate of conversion to multiple myeloma following successful treatment of the primary lesion. 6 Yentis proposed that all cases of SPB represented early multiple myeloma. ${ }^{7}$ The strong similarity between multiple myeloma and the disseminated stage of SPB was also noted in an extensive review by Wiltshaw, who supported the hypothesis that SPB represented the same pathological process as However, one was of an IgD subtype and 

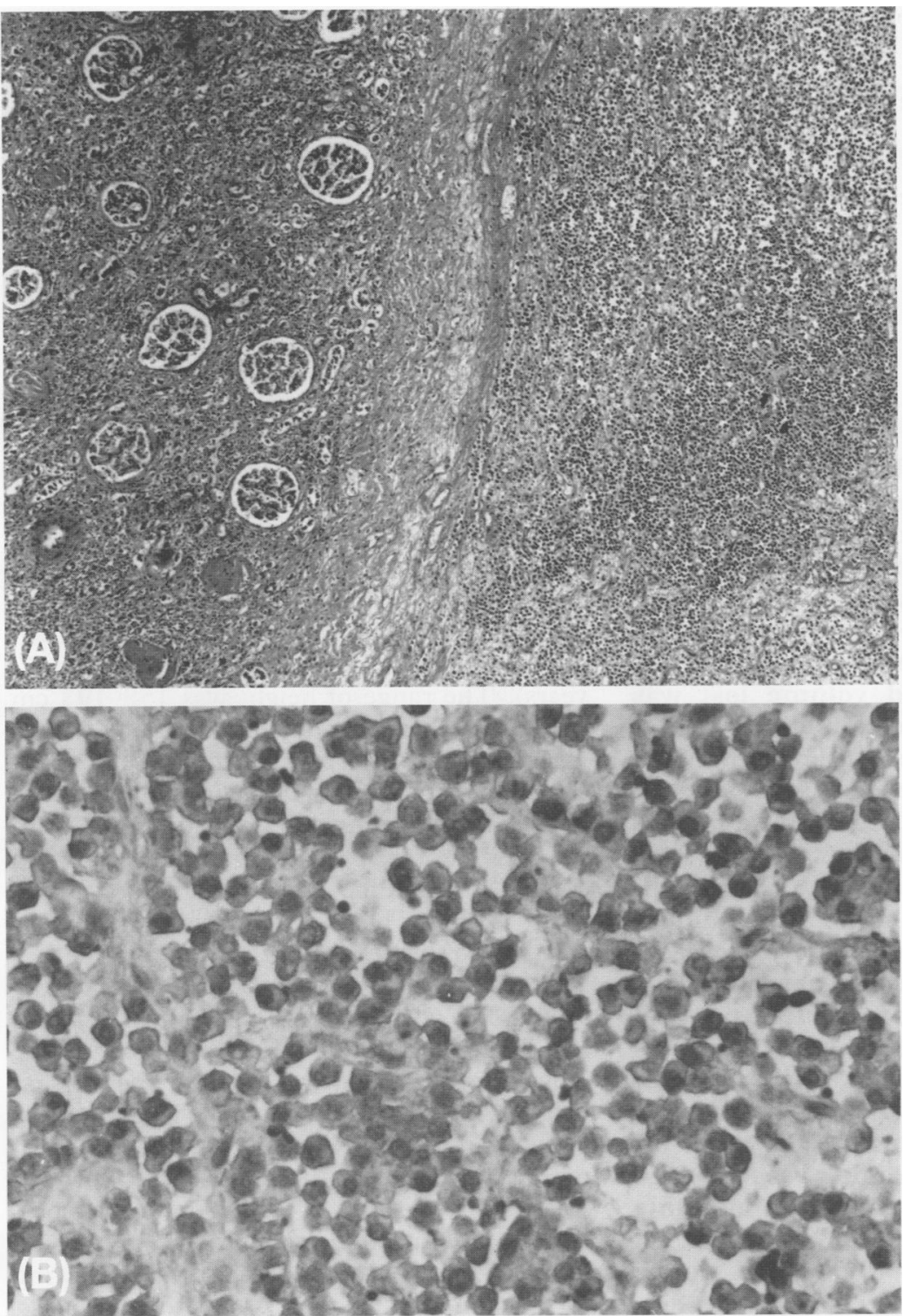

Figure 2 (A) Low power view of tissue obtained from right kidney at post mortem examination showing diffuse infiltration by tumour cells (haematoxylin and eosin). $(B)$ High power view of same tissue as in $(A)$. The tumour cells show plasmacytoid features and $\lambda$ light chain restriction (immunoperoxidase stain). multiple myeloma. ${ }^{5}$ Although our case demonstrated radiological evidence of extraosseous spread from the vertebrae at presentation, the clinical history was short and we feel it is unlikely that the patient's disease had evolved from an SPB at that site.

The evolution of our patient's disease was unusual in that the pattern of spread following relapse from the plateau phase was similar to that seen in some cases of extramedullary plasmacytoma (EMP) in which relapse is often associated with the event of spread. The development of successive, multiple asynchronous EMP at different localisations has also been described. ${ }^{8}$ However, in her review Wiltshaw considered that the behaviour of EMP reflected a malignant process distinctly different from multiple myeloma or SPB. ${ }^{5}$

At presentation our case demonstrated features that satisfied the generally accepted criteria to establish a diagnosis of multiple myeloma of IgG subtype. However, we believe the atypical clinical course disclosed features not hitherto reported in IgG multiple myeloma and underscores the complexity of the plasma cell dyscrasias.

We thank Dr K M Hunt for allowing us to report this case.

1 Jancelwicz Z, Takatsuki D, Sugai S, Pruzanski W: IgD myeloma. Review of 133 cases. Arch Intern Med 1975; 135:87-93.

2 Hayes DW, Bennett WA, Heck FJ. Extramedullary lesions in multiple myeloma. Review of literature and pathoin multiple myeloma. Review of literature

3 Foucar K, Raber M, Foucar E, Barlogie B, Sandler CM, Alexanian R. Anaplastic myeloma with massive Alexanian R. Anaplastic myeloma with massi

4 Knowling MA, Harwood AR, Bergsagel DE. Comparison of extramedullary plasmacytomas with solitary and multiple plasma cell tumours of bone. $\mathcal{F}$ Clin Oncol 1983; 1:255-62.

5 Wiltshaw E. The natural history of extramedullary plasmacytoma and its relation to solitary myeloma of bone and myelomatosis. Medicine 1976;55:217-38.

6 Corwin J, Lindberg RD. Solitary plasmacytoma of bone vs. extramedullary plasmacytoma and their relationship to multiple myeloma. Cancer 1979;43:1007-13.

7 Yentis I. The so-called solitary plasmacytoma of bone. $f$ Fac Radiol 1957;8:132-44.

8 Bauduer F, Tanguy A, Mandard AM: Plasmacytoma with asynchronous metastasis. Immunological and ultrastructural contribution. Arch Anat Cytol Pathol 1992;40:23-7. 